\title{
Corrigendum: Toll-like receptor-2 has a critical role in periodontal pathogen-induced myocardial fibrosis in the pressure-overloaded murine hearts
}

Makoto Kaneko, Jun-ichi Suzuki, Norio Aoyama, Ryo Watanabe, Asuka Yoshida, Yuka Shiheido, Yuichi Izumi and Mitsuaki Isobe

Hypertension Research (2017) 40, 212; doi:10.1038/hr.2016.148

Correction to: Hypertens Research (2017) 40, 110-116; doi:10.1038/ hr.2016.117; Published online 1 September 2016

After publication of this Article, the authors noticed an error in 'Toll-like receptor-2 has a critical role in periodontal pathogeninduced myocardial fibrosis in the pressure-overloaded murine hearts' in the Affiliations section. '1. Department of Periodontology, The University of Tokyo, Tokyo, Japan' was incorrect. The correct affiliation was '1. Department of Periodontology, Tokyo Medical and Dental University, Tokyo, Japan'.

This correction does not affect the results or the conclusions of this manuscript. 